\title{
L'exofiction au service de la réalité dans Merci pour ce roman de Guillaume Prévost
}

\author{
Howayda ISMAIL \\ Maitre de conférences au Département de langue de littérature \\ françaises et de traduction simultanée, Faculté des Sciences Humaines, \\ Université Al-Azhar des jeunes filles
}




\section{Abstract:}

\section{Howayda ISMAIL,}

Associate Professor in the Department of French Literature and Simultaneous Translation, Faculty of Human Sciences, Al-Azhar University of Girls.

\section{Exofiction in the service of reality in Merci for this novel of Guillaume Prévost}

The exofiction is the writing of a fiction based on real elements, authorizing the staging under their own name of characters having existed. According to Frédérique Roussel, the term was coined in 2013 by Philippe Vasset: "The fiction today is built a lot from enigmas that presents the real. He calls this form "exofiction", exactly the opposite of autofiction.

This new trend of writing that comes back with each literary comeback, trying to find a place on the shelves of booksellers for three or four years, pushes us to reflect on the limits that could exist between reality and fiction.

During his presidential campaign in 2012, François Hollande promised the final shutdown, at the end of 2016, of the oldest nuclear power station in France, that of Fessenheim in Haut-Rhin. Indeed, the closure of this plant guaranteed him the support of the ecologists. So, on the scheduled date, and to keep his promise, the President of the Republic decides to go to the site and give a closing speech. Unfortunately, during this presidential visit to Fessenheim, a gas leak took place, which caused unease to the President. Three days later, François Hollande wakes up in Mulhouse, in the shoes of Michel Gravier, a modest worker at the nuclear power plant. And conversely, Michel Gravier, also wakes up, to find himself in a room in Val-de-Grace and in the skin of François Hollande.

Through this fictitious situation of exchange or substitution, imagined by Guillaume Prévost in Merci for this novel, a book published in September 2016, this one paints to its readers a critical table behind the scenes of the Elysée and the politics of François Holland. Which leads us to ask ourselves the following question: To what extent can the exofiction be a critical instrument of value?

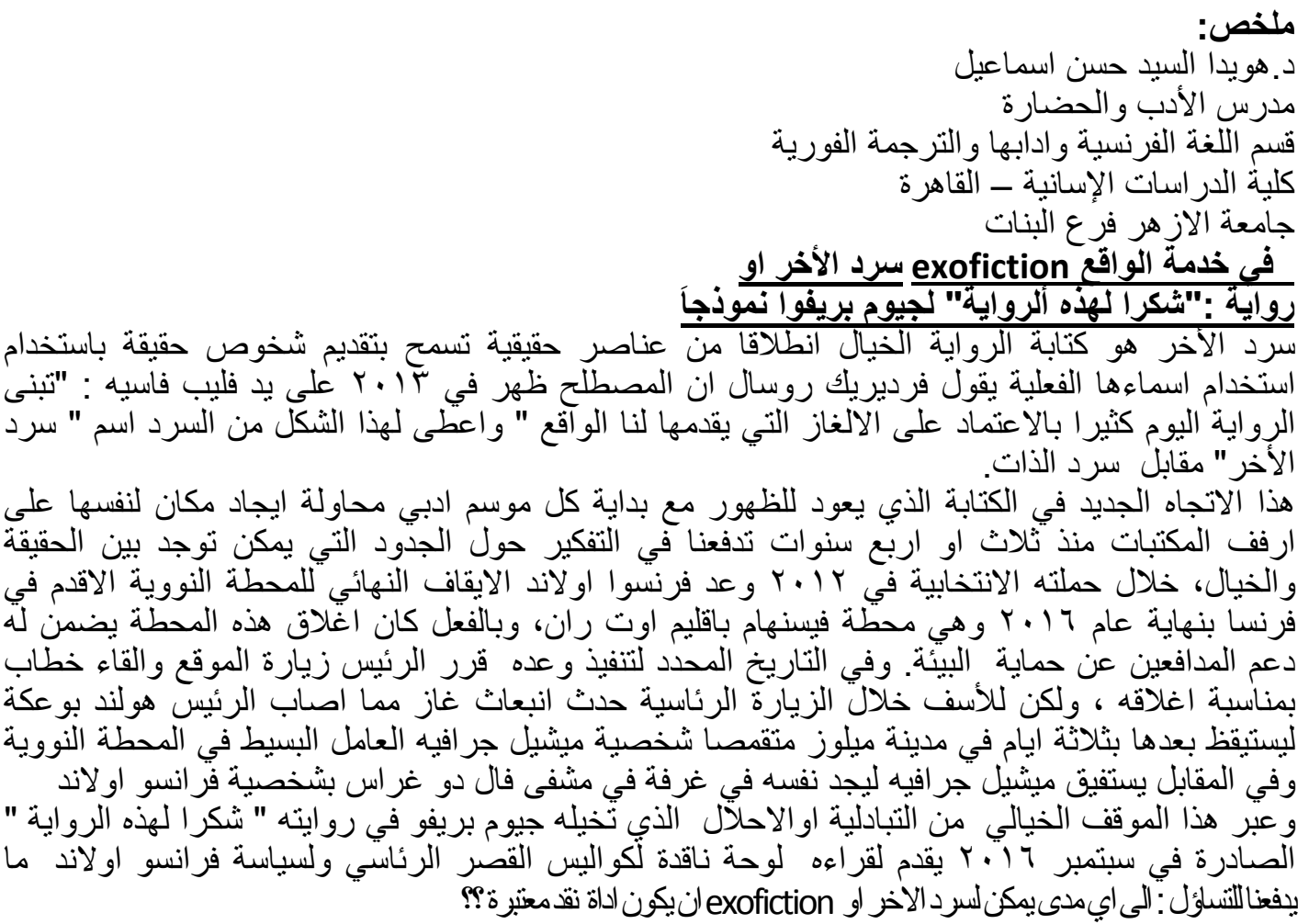


L'exofiction est l'écriture d'une fiction à partir d'éléments réels, en s'autorisant la mise en scène sous leur propre nom de personnages ayant existé. Selon Frédérique Roussel, le terme a été forgé en 2013 par Philippe Vasset : "La fiction aujourd'hui se construit beaucoup à partir d'énigmes que nous présente le réel. "Il baptise cette forme « exofiction », exactement l'inverse de l'autofiction. ${ }^{1}$

$\mathrm{Au}$ cours de ces dernières années, les ouvrages d'exofiction se multiplie d'une rentrée littéraire à l'autre. Ainsi, nous pouvons citer quelques exemples de cette nouvelle tendance tel l'ouvrage de Yasmina Khadra, La Dernière Nuit du Raïs sur Mouammar Kadhafi, paru chez Julliard en 2015 et celui de Bernard Chambaz, Vladimir Vladimirovitch sur Vladimir Poutine, paru chez Flammarion la même année. En 2017, Jean-Pol Hecq publie son ouvrage Tea time à New Delhi sur Che Guevara et Indira Gandhi aux Editions Luce Wilquin. Cependant, il est à préciser que l'exofiction ne se limite pas aux chefs d'État et aux hommes politiques. En 2014, Bye Bye Elvis de Caroline De Mulder, sur Elvis Presley est publié aux éditions Actes Sud. De même, l'ouvrage intitulé La Septième Fonction du langage sur Roland Barthes de Laurent Binet est publié aux édition Grasset en 2015.

Cette nouvelle tendance d'écriture qui revient avec chaque rentrée littéraire, essayant de se trouver une place sur les étagères des libraires depuis quatre ou cinq ans, nous pousse à réfléchir sur les limites qui pourraient exister entre la réalité et la fiction.

Lors de sa campagne présidentielle en 2012, François Hollande avait promis l'arrêt définitif, fin 2016, de la centrale nucléaire la plus ancienne de France, celle de Fessenheim au Haut-Rhin. En effet, la fermeture de cette centrale lui garantissait le soutien des écolos. Ainsi, à la date prévue, et pour tenir sa promesse, le Président de la République décide de se rendre sur le site et prononcer un discours de fermeture. Malheureusement, lors de cette visite présidentielle à Fessenheim, une fuite de gaz a eu lieu, ce qui a causé un malaise au Président. Trois jours plus tard, François Hollande se réveille à Mulhouse, dans la peau de

\footnotetext{
${ }^{1}$ VASSET, Philippe, cité par Frédérique ROUSSEL, « De passage secret », Libération, n 10039 , 23 août 2013, [en ligne], consulté le 27 décembre 2017, URL : http://next.liberation.fr/livres/2013/08/22/philippe-vasset-de-passage-secret 926385
} 
Michel Gravier, modeste ouvrier de la centrale nucléaire. Et inversement, Michel Gravier, se réveille, lui aussi, pour se trouver dans une chambre à Val-de-Grace et dans la peau de François Hollande.

À travers cette situation fictive d'échange ou de substitution, imaginée par Guillaume Prévost dans Merci pour ce roman, ouvrage publié en septembre 2016, celui-ci peint à ses lecteurs un tableau critique des coulisses de l'Elysée et de la politique de François Hollande. Ce qui nous mène à nous poser la question suivante : Dans quelle mesure l'exofiction peut être un instrument critique de valeur?

Nous répondrons à cette question en deux temps : d'abord, en étudiant la période du mandat présidentiel de François Hollande, de 2012 à 2017 avec les problèmes insurmontables : chômage, déclassement et surtout l'insatisfaction du peuple français. Ensuite, dans un deuxième temps : les critiques adressées au président de la République et la part de réalité dans Merci pour ce roman.

Nous sommes face à un roman fictionnel qui nous introduit massivement dans le monde politique avec tous ses méandres. 


\section{La politique de François Hollande à travers le pseudo-Gravier}

Dans le cadre de sa campagne présidentielle, François Hollande cherchait à gagner de la popularité parmi les écolos. Ainsi, pendant le débat de l'entre-deux tours, il s'engage à fermer la centrale nucléaire de Fessenheim. Cet engagement qui figure à la page 28 de son programme, constitue l'événement clé de Merci pour ce roman. "Du coup, il avait choisi de frapper fort : se rendre sur le lieu même de la discorde et prononcer un discours de fermeté ${ }^{2}$. Au début du second chapitre, lors de sa présence à la centrale nucléaire, une fuite de gaz cause un malaise au Président qui se réveille, trois jours plus tard, dans la peau d'un certain Michel Gravier, modeste employé de Fessenheim. Il n'arrive pas à se rappeler de ce qui s'est passé au cours de sa visite : "Il ne distinguait plus qu'un seul visage parmi le public, celui de Michel Gravier. Michel Gravier qui avançait vers lui, le poing levé. Ensuite, le trou noir... $»^{3}$, écrivit Prévost. Mais, petit à petit, la mémoire commence à lui revenir. Il se souvient qu' "Il avait promis la fermeture de la centrale en 2012 et n'avait cessé d'en repousser l'exécution. 2016, 2017... C'était son truc, ça, repousser. En politique, il l'avait appris très tôt, décider revenait à faire des mécontents ou des ingrats. Surseoir vous assurait au contraire des obligés $»^{4} \mathrm{Ce}$ qui était tout à fait vrai puisque quelques mois après son investiture, en septembre 2012, pendant 1'ouverture de la conférence environnementale, le Président déclare la fermeture de la centrale en fin de l'année 2016. Promesse qui d'ailleurs n'a jamais été tenue. Par ce début de roman, très symbolique, l'auteur envoie un message très important aux lecteurs du roman. Il s'agit de mettre l'accent sur un point faible majeur parmi ceux qui ont contribué à faire chuter la popularité de l'ex-président : celui de ne pas tenir ses promesses.

\footnotetext{
${ }^{2}$ PRÉVOST, Guillaume, Merci pour ce roman (French Edition) (Emplacements du Kindle 131). Éditions François Bourin. Édition du Kindle.

${ }^{3}$ Ibid, (Emplacements du Kindle 139).

${ }^{4}$ Ibid, (Emplacements du Kindle 120-123).
} 
François Hollande était connu comme le président incapable de tenir ses promesses. Sur les 189 promesses qu'il avait annoncées dans son programme, lors de sa campagne pour les présidentielles 2012, 72 seulement ont été tenues ${ }^{5}$, ce qui désigne environ $\mathbf{3 8 \%}$ des promesses. Et voilà qu'il le déclare lui-même, lorsqu'après l'incident, dans la maison de Gravier, il était en train de recenser les tâches urgentes à accomplir : "déclarer ses cartes de crédit volées pour obtenir de nouveaux codes et financer à la fois le concert des Bankruptcy - une promesse au moins qu'il s'efforcerait de tenir ». ${ }^{6}$ Promesse qu'il avait faite à la fille de Gravier qui lui avait prêté son ordinateur pour regarder les vidéos de la visite. Il n'est certainement pas question de faire l'inventaire des engagements que le candidat Hollande avait fait aux citoyens français lors de sa campagne. Cependant, nous allons mentionner ceux qui touchaient de près à la vie quotidienne du peuple.

Tout d'abord, nous mentionnerons une promesse que beaucoup de Français tenait à cœur, celle de la discrimination. Le candidat « socialiste » à la présidentielle en 2012 s'était engagé devant son électorat de "Lutter contre toute discrimination au logement et à l'embauche $»^{7}$ Or, le 15 février 2013, Le Monde publie une tribune où plusieurs personnalités appellent François Hollande à tenir cette promesse « oublié » de lutte contre les discriminations. ${ }^{8}$ Guillaume Prévost le fait entendre à ses lecteurs par l'intermédiaire de son héros : "C'était l'inconvénient des promesses : on ne regrettait jamais tant de les avoir faites qu'au moment de les tenir ${ }^{9}$, déclare-t-il. En avril 2014, une étude publiée par l'Insee $^{10}$ a révélé que les discriminations persistent en France. Elle souligne deux points très importants : d'une part, il existe en moyenne deux ans et demi pour l'accès au logement, entre les ménages d'origine européenne et les ménages d'origine non européenne. D'autre part, la même étude, souligne qu'avec un CV identique, les candidats

\footnotetext{
${ }^{5}$ LUI PRÉSIDENT, Les promesses de François Hollande passées au crible, [Site en ligne], consulté le 31 juillet 2018, tiré de https://www.luipresident.fr/francois-hollande/60-engagements ${ }^{6}$ PRÉVOST, Guillaume, Op. cit. (Emplacements du Kindle 375-377). ${ }^{7}$ Ibid, p. 21

8 «La lutte antidiscrimination ou la promesse oubliée », Le Monde, Tribune, publié le 15 février 2013, [en ligne], consulté le 15 juin 2017, https://www.lemonde.fr/idees/article/2013/02/15/la-lutteantidiscrimination-ou-la-promesse-oubliee 1833332 3232.html

${ }^{9}$ PRÉVOST, Guillaume, Op. cit. (Emplacements du Kindle 1598).

${ }^{10}$ Insee : Institut national
} 
« issus de l'immigration » ont $40 \%$ de chances de moins de trouver un emploi. En plus, le ministre du travail, François Rebsamen, en mai 2015, ajoute la cerise sur le gâteau en enterrant le $\mathrm{CV}$ anonyme, estimant que celui-ci pouvait nuire à la productivité. ${ }^{11}$

Ensuite, «Faire construire 2,5 millions de logements intermédiaires, sociaux et étudiants, dont 150000 logements très sociaux». Cet engagement figurait à la page $18 \mathrm{du}$ programme de François Hollande. Faute de budget, sur les cinq années, seuls 1,7 millions de constructions ont commencé. Donc, l'objectif n'a pas pu être atteint.

Enfin, "Introduire une tarification solidaire dans les transports, fondée sur le quotient familial de l'usager $»{ }^{12}$ Cet engagement consiste à fixer le tarif d'un abonnement de transport en fonction des revenus d'un foyer. Hormis les villes pionnières telles Strasbourg, Caen, Dunkerque, Nantes et d'autres villes qui avaient mis en place un système de tarification solidaire bien avant le début du quinquennat, l'engagement a été brisé.

Revenons maintenant à Mulhouse, pourquoi fermer Fessenheim ? Cette centrale nucléaire, la plus ancienne de France, constituait-elle un vrai danger ? En réalité, Hollande savait bien qu' "II n'y aurait ni catastrophe majeure ni krach économique. L'important, c'était le symbole. Et l'alliance avec les Verts. Cécile Duplo [...] n'avait cessé de le bombarder de textos comminatoires : pas de fermeture, pas de soutien des écolos. Et pas de soutien des écolos, pas de renouvellement du bail... " ${ }^{13}$ Guillaume Prévost évoque, dans cette partie, Cécile Duflot, la députée de la sixième circonscription de Paris issue du parti «Europe Écologie Les Verts" (EÉLV). De même, il soulève la volonté de François Hollande, lui-même, de se représenter à la fin de son premier mandat. D'ailleurs, l'exprésident livre le fond de sa pensée lors de son entretien téléphonique avec le journaliste du Haut-Rhin : «En même temps, je crois qu'il [le Président] a tort. Le programme qu'il propose arrive beaucoup trop tôt : il va coûter une fortune au pays, pour

\footnotetext{
${ }^{11}$ AFP, « François Rebsamen enterre le CV anonyme », Le point, publié le 19/05/2015, [en ligne], consulté le 31 juillet 2017, tiré de https://www.lepoint.fr/societe/francois-rebsamen-enterre-le-cvanonyme-19-05-2015-1929465 23.php

12 LUI PRÉSIDENT, 15 è des 20 engagements de François Hollande pour les quartiers et territoires délaissés, le 27 mars 2012, [en ligne], consulté le 30 juin 2017, https://www.luipresident.fr/francoishollande/engagement/tarification-solidaire-dans-transports-431

${ }^{13}$ PREVOST, Guillaume, Op. cit., (Emplacements du Kindle 125-128)
} 
ce qui est du démantèlement des centrales comme des investissements dans le renouvelable. [...] Le seul bénéfice qu'il peut en tirer est un bénéfice politique, et encore, à court terme ${ }^{14}{ }^{14}$, ajout-t-il.

Cécile Duflot, celle qui avait occupé le poste de Ministre du Logement et de Légalité des territoires pendant deux ans de 2012 à 2014 ne sera pas la seule personne réelle mentionnée par Prévost. Les lecteurs verront défiler tout au long du roman toutes les personnes qui entouraient François Hollande sous des pseudo-noms. Ainsi, nous rencontrons ses premier-ministres Jean-Marc Zéro et Manuel Balls, ses ministres Darkozy, Ségoline et aussi les deux femmes qui avaient marqué son quinquennat Valkyrie et Jolie Gayet, ainsi que beaucoup d'autres personnes sans oublier Farine Le Pêne sa rivale de droite.

Ainsi, et après cet incident à Fessenheim, l'ex-président se trouve obligé de s'adapter à un corps qui lui est étrange, de vivre, au sein d'une famille dysfonctionnelle, menacé par le chômage et le déclassement. Vivait-il un cauchemar? ou "Un juge suprême, là-haut, qui au terme de son mandat avait estimé qu'il était temps pour lui d'expier ses fautes. De subir ce qu'il avait fait subir à ceux qu'il gouvernait » ${ }^{15}$, reconnaît-il. Cette déclaration faite par le pseudo-Gravier fait revenir aux lecteurs les leçons de morales données par La Fontaine à travers ses fables. Le moment est arrivé pour que celui qui, depuis quatre ans fait souffrir son peuple, soit puni. Qui est-ce « Michel Gravier» dont il occupe le corps ? C'est un travailleur issu de la classe ouvrière, qui pendant de longues années avait souffert pour subvenir aux besoins de sa famille et qui risque finalement, à l'âge de quarante ans et à cause des décisions d'un président hésitant, de se trouver sur les listes des chômeurs de PôleEmploi. Quel hasard ! Dans la réalité, inverser la courbe du chômage, n'était-il pas l'enjeu majeur du mandat de François Hollande ? Il avait même demandé à être jugé sur cette promesse à la fin de son mandat. Faire face au problème du chômage était la priorité numéro 1 des Français, la bataille que François Hollande n'a pas pu remporter. Depuis son

\footnotetext{
${ }^{14}$ Ibid., (Emplacements du Kindle 432-437).

${ }^{15}$ PREVOST, Guillaume, (Emplacements du Kindle 330-331).
} 
élection et tout au long de son quinquennat, le président n'a pas réussi à faire baisser le taux du chômage. Malgré la baisse du coût du travail grâce au Pacte de responsabilité, malgré les contrats aidés et les primes aux très petites entreprises (TPE) ainsi que la réforme du code du travail, 97\% des Français, estimaient que le président a "plutôt échoué" en matière d'emploi. ${ }^{16}$ Toutefois, l'Institut national de la statistique et des études économiques (Insee) apporte un bilan moins pessimiste en constatant un léger repli du chômage fin 2013. Selon France 24 à cette époque, "François Hollande affiche une cote de popularité historiquement basse, avec seulement $12 \%$ d'opinions favorables ${ }^{17}$. S'il faut juger sur la totalité du mandat, les résultats ne seront pas non plus en faveur du président. En fait, Hollande avait pris ses fonctions en mai 2012, au moment où Pôle emploi comptait 4356 000 chômeurs des trois catégories A, B et C; vers la fin du mandat, en mars 2017, les chiffres publiés étaient de 5503800 chômeurs pour les trois catégories. Ce qui veut dire que le taux de chômage avait augmenté donc de $26 \% .{ }^{18}$ Un point fort pour Farine Le Pêne sa rivale de droite: "Un million de chômeurs de plus sous Darkozy, un autre sous Hollande, vous pensez que l'UMPS fera mieux ensuite ? ${ }^{19}$, lui fait remarquer une blonde du rassemblement de droite.

En effet, de 2012 à 2017, Hollande fit face à plusieurs problèmes insurmontables au cours de son mandat. Il n'a pas pu maintenir les promesses qu'il avait mentionné dans son programme lors de sa campagne présidentielle. Ceci favorise son impopularité et le pousse finalement à renoncer à se présenter pour un second mandat. Ce qui constituait une grande première dans l'histoire de la $\mathrm{V}^{\mathrm{e}}$ République ! Depuis 1958, tous les prédécesseurs de Hollande s'étaient représentés à l'issue de leur premier mandat, exception faite pour Georges Pompidou, décédé pendant son septennat en 1974. À la fin de son allocution

\footnotetext{
${ }^{16}$ Selon un sondage publié lundi 3 novembre 2014 par Odoxa

${ }_{17}$ Anonyme, François Hollande : Les cinq raisons d'une impopularité record, le 06/11/2014, [en ligne], consulté le 26 février 2017, tiré de https://www.france24.com/fr/20141106-francoishollande-plus-bas-sondages-television-interview-impopularite-president-france

18 Eléa POMMIERS et Maxime DELRUE, «Emploi : Les modestes résultats du quinquennat Hollande », Le monde, le 27 avril 2017, [en ligne], consulté le 26 juin 2017, tiré de

https://www.lemonde.fr/les-decodeurs/article/2017/04/27/emploi-et-chomage-le-mediocre-bilandu-quinquennat-hollande 5118948 4355770.html

${ }^{19}$ PREVOST, Guillaume, (Emplacements du Kindle 1030).
} 
télévisée d'une dizaine de minutes prononcée depuis le palais de l'Élysée, il déclare le premier décembre 2016: "J'ai décidé de ne pas être candidat à la présidentielle, au renouvellement de mon mandat». Vu les problèmes rencontrés, il semble, réellement, que le Président sentait qu'il n'avait aucune chance d'être réélu en mai 2017 et a voulu se retirer pour éviter un échec électoral. Quels sont donc ces problèmes qui obligèrent Hollande de se retirer de la scène politique?

Voilà qu'à travers cette situation assez drôle, l'auteur de Merci pour ce roman va faire vivre au Président la vie modeste de la classe ouvrière lorsqu'il entre avec sa femme dans un supermarché à bas prix, «[...]. Lui était fasciné par cet étalage brutal de produits, à même les cartons, à même le sol, ces conditionnements de régiment [...], ces marques improbables, ce choix délibéré de couleurs criardes et d'affiches suggestives jusqu'à l'écourement. Voilà qui expliquait la qualité très relative de la cuisine de la maison Gravier. [...] Était-ce à cela que son quinquennat avait réduit les Français ? $»^{20}$ En fait, Guillaume Prévost dénonce les points faibles du quinquennat Hollande à travers Hollande lui-même. En lisant ce roman, le lecteur a l'impression qu'il s'agit d'une sorte d'autocritique. Un peu plus loin, une autre remarque concernant les fichiers trouvés sur l’ordinateur de Michel Gravier, très symbolique d'ailleurs, "des feuilles de compte - qui confirmèrent que le ménage Gravier était en déficit chronique, ce en quoi au moins il était exemplaire du pays $"{ }^{21}$ Prévost fait allusion au problème du déclassement ainsi qu'au problème du chômage. Un peu plus loin, l'auteur ne ratera pas l'occasion pour évoquer aussi le déficit budgétaire dans lequel plongea la France à la fin du quinquennat de l'ancien Chef de l'État : "Nous n'avons même plus de monnaie et on voudrait nous faire croire qu'on aurait une politique économique ? $\aleph^{22}$, déclara une blonde du rassemblement de Droite.

Outre le problème du chômage, l'économie qui tournait toujours au ralenti compromet le quinquennat du président. Même si la croissance économique du pays devait s'améliorer en 2015 pour afficher 1\%, celle-ci n'a pu réaliser que $0,3 \%$ en 2013 et 0,4\% en

\footnotetext{
${ }^{20} \mathrm{Ibid}$, (Emplacements du Kindle 700-706).

${ }^{21}$ Ibid, (Emplacements du Kindle 975).

${ }^{22}$ PREVOST, Guillaume, (Emplacements du Kindle 1082).
} 
2014. Des résultats très modestes qui, en effet, plaçaient la France au $15^{\mathrm{e}}$ rang sur les 18 pays de la zone euro.

De plus, un autre problème venait compromettre le quinquennat de François Hollande, c'est celui de la dette publique. Malheureusement, le président n'est pas parvenu à baisser la dette publique de la France. Celle-ci a continué à augmenter impitoyablement pour passer de 1868 milliards d'euros en 2012 (89,5\% du PIB) à 2147,2 milliards d'euros au 31 décembre 2016, soit 96\% du PIB. ${ }^{23}$ De même, le 29 juin 2017, la Cour des comptes, dans son audit des finances, accuse le gouvernement sortant d'avoir manqué de sincérité en constituant son budget. À cet audit, le premier ministre, Édouard Philippe réagit vivement: "Nous héritons d'un dérapage des dépenses de 8 milliards d'euros, c'est inacceptable!» «Tous ces artifices placent la France dans une situation de grande fragilité vis à vis de ses voisins européens. ${ }^{24}$, a-t-il ajouté. Ainsi, nous pouvons constater que le problème de la dette publique constitue encore un point noir qui vient s'ajouter à la liste pour discréditer le président.

Ajoutons au problème de la dette publique celui du choc fiscal. En réalité, l'arrivé des socialistes au pouvoir en 2012 se traduisit par une augmentation excessive des prélèvements fiscaux. À en croire Éric Verhaeghe, énarque et ancien président de l'Association pour l'emploi des cadres (APEC), cette augmentation représente «En 4 ans, [...] plus de 1.000 euros par Français et par an qui sont engloutis dans la dépense publique, sans que ces Français ne mesurent concrètement quelle amélioration du service est proposée en contrepartie ${ }^{25}$ L'impact ne tarda pas à se faire sentir sur 1'emploi. Dans les années 2010 - 2011, l'économie française était sur la bonne voie pour la création

\footnotetext{
${ }^{23}$ Selon les statistiques publiées par l'Insee

${ }^{24}$ Elena Scappaticci, «Audit de la Cour des comptes : Philippe juge « inacceptable » le dérapage des dépenses », Le Figaro, le 30/06/2017, [en ligne], consulté le 26 août 2017, tiré de http://www.lefigaro.fr/conjoncture/2017/06/29/20002-20170629ARTFIG00194-audit-de-la-courdes-comptes-sapin-et-eckert-se-justifient.php

${ }^{25}$ Éric Verhaeghe, « Fiscalité : une autre histoire du quinquennat Hollande » », Contrepoints, publié le 12 octobre 2016, [en ligne], consulté le 26 août 2017, tiré de https://www.contrepoints.org/2016/10/12/266654-fiscalite-croissance-histoire-quinquennathollande
} 
d'emploi. Or, les trois premières années du quinquennat Hollande, deux cent mille emplois furent détruits et les sept cent mille nouveaux arrivants sur le marché du travail se trouvèrent accablés par le chômage et le déclassement.

Par ailleurs, «inverser la courbe du chômage n'était pas le seul pari auquel le président devait faire face. Sa présidence connaît plusieurs autres difficultés d'ordre politique, notamment avec les protestations concernant la loi Travail portée par la ministre Myriam El Khomri au début de l'année 2016. Les manifestations entreprises par le mouvement social "Nuit-Debout » révèlent les lacunes du système politique français à cette époque. D’ailleurs, Guillaume Duval, journaliste français, explique bien ce phénomène : "Ces grands mouvements de protestation à répétition ont assez largement une spécificité hexagonale, qui intrigue nos voisins. Même s'ils laissent des souvenirs forts à ceux qui les vivent, ils sont malgré tout aussi le signe récurrent d'un dysfonctionnement majeur de la société française $[. ..] \Perp^{26}$. En effet, l'impact de ses manifestations influence négativement sur la popularité de François Hollande. En juin 2016, elle est au plus bas depuis son élection avec seulement $18 \%$ d'opinions positives. Il faut ici mentionner que le niveau le plus bas pour Jacques Chirac avait été de 32\% en mai 2006, vers la fin de son second mandat et de 30\% pour Sarkozy en 2011. Ce qui veut dire que François Hollande, avec ce score, a battu les records de ces prédécesseurs et s'est classé au rang du président le plus impopulaire de la Vépublique.

Malheureusement les choses ne s'arrêteront pas là. Un autre problème grave vint peser sur le quinquennat présidentiel : celui du terrorisme. En effet, le mandat Hollande a été marqué par des attentats de masse, à Paris en janvier et novembre 2015 ou à Nice en juillet 2016. "Et puis il y avait eu les Événements... Le massacre réinventé des Innocents. Les balles contre les sourires, la joie fauchée dans le sang, le meilleur de l'homme anéanti par le pire ", ${ }^{27}$ écrivit Prévost. C'est en essayant de réagir face au terrorisme que François Hollande se mit dans une situation très critique lorsqu'il proposa la déchéance de la

\footnotetext{
${ }^{26}$ Guillaume DUVAL, "Debout ", Alternatives économiques, le 01/05/2016, [en ligne], consulté le 26 février 2017, tiré de https://www.alternatives-economiques.fr/guillaumeduval/debout/00011012
}

${ }^{27}$ PREVOST, Guillaume. Op. cit. (Emplacements du Kindle 344). 
nationalité. Proposition qui fut décrite ironiquement par l'auteur comme étant «un manque de lucidité regrettable, presque une déchéance de rationalité... Et qui n'avait rien empêché, de surcroît $»^{28}$ D'ailleurs Hollande regrette sa proposition lors de son discours du premier décembre 2016 au palais de l'Élysée : «Je n'ai qu'un seul regret (...), c'est d'avoir proposé la déchéance de nationalité, parce que je pensais qu'elle pouvait nous unir alors $q u^{\prime}$ elle nous a divisés $»^{29}$, avoue-t-il. Cette proposition causa un vrai malaise à plus de trois millions de Français binationaux qui n’ont rien à faire avec le terrorisme. En plus, c'est dans son propre camp que Hollande sema la contestation la plus vigoureuse. La majorité des socialistes considère que cette réforme mènerait à la discrimination parmi les Français. Quelques mois plus tard, le Président est contraint de renoncer à son projet qui représente, dans le bilan de son quinquennat, un échec parmi les plus sévères.

\section{Les coulisses de l’Élysée à travers Michel Gravier}

Si après l'incident qui eut lieu à la centrale nucléaire, le chef de l'État s'est réveillé dans la peau de Michel Gravier, il fallait donc qu'il se pose des questions sur la personne au pouvoir. Assis devant l'écran, la télécommande à la main, il était étonné par « [...] cette marche du monde qui se poursuivait sans lui : les résultats sportifs, le Proche-Orient, les guerres, les soubresauts de l'économie, les chefs d'État qu'il avait côtoyés, ses propres ministres, les députés de son parti qui ronronnaient leurs phrases toutes faites, indifférents au drame qu'il traversait $»^{30}$. Mais la réponse ne tarda pas à venir, «La conclusion était simple : Michel Gravier dirigeait désormais la France. Un coup d'État de l'intérieur, en quelque sorte ", ${ }^{31}$ écrivit Prévost.

Si le président s'est réveillé à Mulhouse, Michel Gravier, quant à lui, il se réveille dans une chambre à l'hôpital Val-de-grâce. «D'abord, il avait perdu vingt-

\footnotetext{
${ }^{28}$ Ibid., (Emplacements du Kindle 347).

${ }^{29}$ HOLLANDE, François, vidéo " L'intégralité du discours de François Hollande », Le Parisien TV, le $1^{\text {er }}$ décembre 2016, [en ligne], consulté le 26 août 2017, tiré de http://videos.leparisien.fr/video/lintegralite-du-discours-de-francois-hollande-01-12-2016x 5469e1\#xtref=https\%3A\%2F\%2Fwww.google.fr\%2F
}

${ }^{30}$ PREVOST, Guillaume, (Emplacements du Kindle 155-157).

${ }^{31}$ Ibid, (Emplacements du Kindle 368-369). 
cinq centimètres. Ses bras et ses jambes avaient rétréci comme de vieux vêtements au lavage et son ventre sous le tissu ressemblait à un rôti de dindonneau. Il avait l'impression qu'on l'avait amputé des extrémités et rembourré au milieu ${ }^{32}$ II s'étonna de voir au pied de son lit, Manuel Balls, le chef du gouvernement ! Il essayait de comprendre ce qui s'était passé. Là aussi, la réponse ne tarda pas à venir. Michel Gravier, après trois jours de sommeil à l'hôpital Val-de-Grâce, il se réveille dans la peau du président de la République. Si à Mulhouse, il y avait un président qui cherchait un moyen pour retrouver sa vraie identité ; ce n'était pas le cas de Michel Gravier.

Contrairement à Hollande, Michel Gravier, le pseudo-président de la République saute sur l'occasion pour profiter de sa nouvelle chance "Et voilà qu'on lui offrait une deuxième chance. Une nouvelle espérance de vie, même... À lui le sommet de la pyramide, là-haut, tout là-haut, à tutoyer le ciel. On lui proposait d'être Dieu et il aurait refusé ? " ${ }^{33}$, pensait-il. II doit donc s'accrocher. Syndicaliste de cœur, il va se débattre à l'Élysée pour assurer la défense collective et individuelle des salariés du nucléaire. Coup de chance ! II a pu réussir cette mission. C'était lors de son départ pour une visite officielle en Iran, au cours d'un entretien avec la directrice générale de la multinationale française de l'atome : "-Vous réembauchez les gars d'Eden et je décroche votre contrat. - Vous le décrochez comment ? - Je suis en voyage officiel, non ? Elle réfléchissait à toute vitesse, concentrée. - Impossible de reprendre tout le monde, finit-elle par répondre. La survie d'Eden en dépend. Je peux aller jusqu'à la moitié, pas plus... Et seulement à condition que les Iraniens nous confient la centrale, bien sûr $»^{34}$. Guillaume Prévost s'interrogeait-il sur la capacité d'un homme du peuple à jouer le rôle du Président de la République ? Là aussi, il s'agissait indirectement d'une critique faite à François Hollande. Guillaume Prévost reprochait au Président une politique étrangère décevante. II est donc nécessaire de préciser que cette visite n'a jamais eu lieu. Pendant tout son mandat François Hollande n'a pas visité l'Iran et "[...], jamais la France n'a été consultée ni

\footnotetext{
${ }^{32}$ Ibid, (Emplacements du Kindle 243).

${ }^{33}$ PREVOST, Guillaume, (Emplacements du Kindle 496-498).

${ }^{34}$ Ibid., (Emplacements du Kindle 1205-1210).
} 
impliquée par les américains sur l'accord sur le nucléaire iranien. Nous avons été au mieux un spectateur attentif ${ }^{35}$. Ce qui explique l'idée de Prévost d'avoir tournée la visite en situation anecdotique.

Cependant, Gravier devait faire face à d'autres défis. Ceux de la vie privée de l'exprésident. Le sujet qui a fait couler beaucoup d'encre tant au niveau national qu'au niveau international. Peu après son réveil, avant de quitter l'hôpital, au cours d'un entretien avec le chef du gouvernement, ce dernier lui dit : "Il y a deux choses dont je dois te parler en privé, François. Deux choses qui, malheureusement, ne peuvent pas attendre. D'abord, à propos de Valkyrie: le tournage de Merci pour ce moment a commencé. Elle a pas mal hésité mais elle a fini par dire oui... On a pu se procurer le script et crois-moi, il est sanglant. Bien plus que le bouquin $»^{36}$. Prévost évoque ici une affaire qui, bien que personnel, faisait du président français la cible de toutes les attaques. Il s'agit de la première dame de France, l'ex-compagne du président, Valérie Trierweiler. En fait, la séparation du couple est intervenue lorsque les journaux français avaient révélé la liaison du président avec l'actrice Julie Gayet. Il est clair que cette séparation n'était pas facile pour Trierweiler, ce qui l'incita en 2014 à publier un ouvrage qu'elle a intitulé Merci pour ce moment où elle régla ses comptes avec François Hollande. En effet, Trierweiler a rapporté dans son livre tous les propos injurieux de Hollande contre les pauvres. Elle le dépeint comme quelqu'un qui méprise les pauvres en les qualifiant de «sans dents». L'expression, ayant choqué tous les internautes de gauche comme de droite, a été reprise maintes fois sur les réseaux sociaux. Par la suite, cette expression, stigmatisant les pauvres, devient tellement populaire qu'elle fut utilisée dans certaines publicités. De plus, cette expression est intégrée dans le langage courant des médias, des journaux ou même de la radio, pour désigner les pauvres. Trierweiler le qualifie aussi de «quelqu'un de froid,

\footnotetext{
35 Olivier Odibert-Troin, " Migrants : La triple erreurs de François Hollande », Economie Matin, publié le 11/09/2015, [en ligne], consulté le 31 juillet 2018, tiré de http://www.economiematin.fr/news-migrants-francois-hollande-crise-politique
}

${ }^{36}$ PREVOST, Guillaume. Op. cit., (Emplacements du Kindle 295). 
cynique et menteur $»^{37}$. Sans aucun doute, cet ouvrage a eu un impact très négatif sur la réputation de Hollande. Il est dédaigné au sein de son propre camp.

François Hollande faisait partie des rares président qui sont arrivés au pouvoir nonmarié. Avant lui, de 1848 à 1852, Louis-Napoléon Bonaparte, unique président de la II ${ }^{\mathrm{e}}$ République, s’installe à l'Élysée en célibataire. Plus tard, en 1924, le Président Gaston Doumergue entre à l'Élysée en célibataire et ne se marie que douze jours avant la fin de son septennat. Quant à Nicolas Sarkozy, il est resté célibataire, pendant trois mois et demi, entre son divorce et son remariage avec la chanteuse Carla Bruni.

Mais François Hollande, le président célibataire, ne s'installe pas seul à l'Élysée. Il y arrive avec Valérie Trierweiler, « la Première Dame ». Or, ce statut, même s'il semblait ne pas déranger les Français, intriguait la majorité des autres chefs d'État. D’ailleurs, aux États Unis comme au Royaume Uni, la situation choquait et l'étonnement, parfois même la moquerie régnaient dans les médias. À cause de cette situation très particulière, lors de la première visite présidentielle de mai 2012 aux États Unis, les médias américains 1'ont baptisée la «first girlfriend ». Ainsi, la chaîne de télévision conservatrice américaine Fox News explique que "divorcée à deux reprises, elle sera la Première dame non mariée à entrer à l'Élysée [...] Shocking! ». ${ }^{38}$ De même, lorsqu'elle a voulu accompagner le Chef d'État lors de son voyage officiel en Inde, en février 2014, l'Élysée a dû négocier auprès des responsables du protocole indien, très strict, l'autorisation pour cette «première compagne de France » d'être aux côtés du président durant la visite. Or six ans plus tôt, en janvier 2008, Carla Bruni avait dû renoncer à accompagner Nicolas Sarkozy en Inde, puisqu'ils n'étaient pas encore mariés. Par contre, dans certains autres États, Valérie Trierweiler n'était pas la bienvenue. De ce fait, il n'était pas prévu qu'elle accompagne le président français au cours de sa visite au Vatican.

\footnotetext{
${ }^{37}$ TRIERWEILER, Valérie, Merci pour ce moment, Éditions des Arènes, version numérique en format PDF, Paris, 2014, p. 229

${ }^{38}$ SLATE.FR, «Seulement en France », Hollande, Royal, Trierweiler et la presse étrangère, publié le 12 mai 2012, [en ligne], consulté le 30 juin 2017, tiré de https:/www.slate.fr/lien/54905/hollande-royaltrierweiler-presse-etrangere
} 
Si l'auteur du roman se servait, d'une part, de son Hollande pour dénoncer certaines lacunes à travers l'autocritique ; d'autre part, il se servirait de son Gravier pour révéler aux lecteurs ce qui se passe, à l'intérieur de l'Élysée ainsi que les échecs de François Hollande à diriger le pays, notamment en ce qui concerne la politique étrangère. Nous le trouvons lors d'une émission, incapable de répondre aux questions qui lui sont posées concernant la rencontre avec son homologue iranien, et « Dans le studio, tous les regards étaient braqués sur lui [...], et, partout dans le pays, des inconnus qui trempaient leur pain dans le café en se demandant comment ils avaient pu élire ce guignol cinq ans plus tôt. ${ }^{39}$

Nous constatons alors que la politique étrangère du président n’a pas été épargné dans Merci pour ce roman. Prévost profitera de la présence de Gravier au local du rassemblement de Droite pour laisser entendre une remarque très révélatrice concernant I'intervention française au Mali et en Centre Afrique : "Plutôt que de gaspiller l'argent en menant des guerres n'importe où et en laissant entrer chez nous des réfugiés qui n'ont rien à y faire ? ${ }^{40}$ En même temps, Prévost critique clairement la politique migratoire laxiste de François Hollande. D'ailleurs, les erreurs de Hollande en matière de crise des migrants ont été dénoncées par le député de la $8^{\text {ème }}$ circonscription du Var, Olivier Odibert-Troin, dans un article publié en septembre 2015 lorsqu'il écrivit : «Ces trois erreurs historiques du Président Hollande pèseront lourd sur notre capacité d'intégration des peuples martyrs dans notre pays ; [...] et altèreront pour longtemps l'image de la patrie des droits de l'homme à l'étranger $»^{41}$.

Lors de la conférence semestrielle, en novembre 2012, le nouveau Président de la République annonce aux Français qu' "En aucun cas la France n'interviendra seule au Mali », ce qui n'était pas du tout le cas lorsqu'en janvier 2013, François Hollande engage une intervention militaire au Mali. Deux mois plus tard, il promet le 28 mars sur France 2, qu' "Il n'y aura plus qu'un millier de soldats français au Mali à la fin 2013 ». II avait même

\footnotetext{
${ }^{39}$ PREVOST, Guillaume. Op. cit. (Emplacements du Kindle 1745-1748).

${ }^{40} \mathrm{Ibid}$, (Emplacements du Kindle 1090).

${ }^{41}$ Olivier Odibert-Troin, Op. cit.
} 
détaillé un calendrier de retrait des troupes françaises et avait assuré qu'en juillet 2013, il ne resterait que 2000 soldats au Mali. Cependant, en juillet, les soldats français étaient encore 3500. Après le lancement de l'opération militaire Hydre, en novembre 2013, la porte-parole du gouvernement Najat Vallaud-Belkacem a déclaré qu'il allait « sans doute falloir encore renforcer la présence française au Mali $»^{42}$ Le nombre de soldat français au Mali était de 2500 en janvier 2014 lorsque François Hollande annonce un nouveau calendrier de retrait. Le 24 avril 2014, Radio Monte-Carlo (RMC), annonce qu'une nouvelle opération sur un terrain élargi devrait débuter et que des soldats français seraient alors présents au Mali mais aussi au Niger, au Tchad et au Burkina Faso afin de lutter contre le terrorisme. Quelques mois plus tard, lorsque la France s'est engagée dans l'opération Sengaris en Centrafrique, Jean-Yves Le Drian, ministre de la défense, avait parlé d'une courte mission ne dépassant pas six mois. Toutefois, François Hollande déclare le 11 décembre 2013, en conseil des ministres, que les troupes françaises resteront en Centrafrique jusqu'à ce que les troupes africaines prennent le relais. D'ailleurs, même si ces interventions militaires ont été considérées comme un succès pour la France, ils compromettent les promesses faites par le Président. Aussi, l'historien français Roland Lombardi, analyste spécialiste des relations internationales, écrivit dans un article du Figaro: "Pour autant, le bilan de la politique étrangère de la France sous François Hollande peut être considéré globalement comme très décevant ${ }^{43}$

Ainsi, nous réalisons que Guillaume Prévost a pu, en utilisant l'exofiction, décortiquer le quinquennat de François Hollande et mettre en valeur toutes les erreurs de l'ancien Chef de l'État. En quelque sorte, l'auteur a suivi la même voie qui fut suivi au siècle classique par jean de la Fontaine. Le fabuliste avait critiqué continuellement, par l'intermédiaire de ses fables animalières, le pouvoir absolu et injuste de la royauté et avait révélé l'hypocrisie des courtisans qui l'entouraient. De la même

\footnotetext{
${ }^{42}$ AFP, « Vallaud-Belkacem : "sans doute renforcer" la présence militaire française au Mali », L'express, publié le 04/11/2013, [en ligne], consulté le 23 juin 2017 tiré de https://www.lexpress.fr/actualites/1/societe/vallaud-belkacem-il-va-falloir-renforcer-la-presencemilitaire-francaise-au-mali_1296495.html

${ }^{43}$ LOMBARDI, Rolland, «Le piteux bilan diplomatique du quinquennat de François Hollande », Le Figaro, publié le 07/09/2016, [en ligne], consulté le 23 juin 2017 tiré de http://www.lefigaro.fr/vox/monde/2016/09/06/31002-20160906ARTFIG00124-le-piteux-bilandiplomatique-du-quinquennat-de-francois-hollande.php
} 
façon, mais en utilisant une technique différente, Prévost a pu, par l'intermédiaire de sa fable politique, stigmatiser le quinquennat Hollande et déceler ses lacunes.

Enfin, nous nous posons la question: comment l'auteur a-t-il pu exploiter l'exofiction et faire de celle-ci un élément critique de valeur?

L'exofiction est, en réalité, un genre littéraire qui a ouvert une nouvelle voie pour la critique et a permis une nouvelle écriture de l'Histoire. Elle a, en effet, pu renouveler le roman historique. Elle se distingue de la biographie qui relate le réel et du roman historique bâti sur un épisode de l'Histoire, auquel l'auteur mêle des événements et des personnages réels et fictifs. En fait, il s'agit de la réécriture de l'Histoire sous forme de roman littéraire. L'auteur de l'exofiction s'empare d'une personnalité publique pour réécrire complètement son histoire en croisant une partie de la réalité avec une partie de fiction, c'est-à-dire que la réalité se noue à la fiction. Si l'autofiction, procédé utilisé par les auteurs pour mettre sur du papier leurs histoires intimes ainsi que celle de leurs proches, risque d'ennuyer les lecteurs, l'exofiction trouve facilement son chemin vers des lecteurs avides de révélations sur des personnalités célèbres ayant déjà existé.

D'ailleurs, si nous regardons de près le titre du roman, nous constaterons qu'il a déjà existé. Guillaume Prévost a intitulé son roman Merci pour ce roman ce qui nous fait pensé tout de suite à l'ouvrage de Valérie Trierweiler Merci pour ce moment publié dans les éditions Les Arènes, en septembre 2014. Cet essai autobiographique, Trierweiler l'avait consacré à sa relation amoureuse avec François Hollande. Bien que l'auteure de l'ouvrage eût bien expliqué, lors de son interview sur BBC One, que «ce n'est pas une vengeance, ce $n^{\prime}$ est pas une revanche. Ce n'est pas pour le détruire, c'est pour me reconstruire moi »44, il faut dire que ce livre a eu un impact destructeur sur l'image de François Hollande dont la popularité déjà était au plus bas. Si Valérie Trierweiler a saisi l'occasion, à travers son ouvrage, pour régler ses comptes avec François Hollande ; Guillaume Prévost, de son côté,

\footnotetext{
${ }^{44}$ Interview Trierweiler : nouvelles déclarations choc à la BBC [VIDÉO], Mis à jour le 23/11/14, [en ligne], consulté le 31 juillet 2018, tiré de https://www.linternaute.com/actualite/personnalites/1205792interview-trierweiler-nouvelles-declarations-choc-a-la-bbc-video/
} 
a utilisé cette nouvelle tendance d'écriture qu'est l'éxofiction pour dévoiler les points faibles du quinquennat de l'ancien Chef de l'État.

Nous pouvons aussi ajouter que dans cette tendance qu'est l'exofiction, il y a ceux qui trouvent leur bonheur, les avocats !! La rentrée littéraire de 2015 n'avait-elle pas été marqué par le procès d'Irina Ionesco contre Simon Liberati. La photographe l'avait assigné en justice parce que, selon elle, son roman Eva, publié aux Éditions Stock, constituait une atteinte à sa vie privée. Par la suite, elle a été déboutée par le tribunal de grande instance de Paris. Ironiquement, Irina Ionesco fut représentée, par un avocat, lui-même écrivain, éditeur, ayant publié un livre sur les ouvrages censurés et appelant à la liberté d'expression, sous toutes ses formes. Il est à noter que le cas d'Irina Ionesco et Simon Liberati n'était pas le seul de son genre. Nous pouvons aussi citer le cas de Régis Jauffret, de Christine Angot ou encore celui de Patrick Poivre-d'Arvor qui ont subi tous le même sort. Pour chacun des trois auteurs, l'accusation est toujours la même : «Atteinte à la vie privée ». Ce qui nous laisse croire que l'attaque d'ouvrages littéraires semble devenir en vogue depuis quelques années.

Ainsi, à travers ce nouveau genre à la fois documenté et créatif, Guillaume Prévost, dans Merci pour ce roman, a su bien croiser le réel à l'imaginaire. Son François Hollande était un type géant avec "D'immenses mains poilues qui dépassaient d'un pyjama bleu et de grands genoux dessinant d'imposantes collines sous la couverture [...]. II lui fallut une interminable minute pour se convaincre qu'en vérité elles lui appartenaient " ${ }^{45}$ Même s'il a gardé son vrai nom "François Hollande » et son poste " Président de la République », le personnage relève de la fiction. Ce n'est qu'un personnage façonné par l'auteur. Quant à tous les autres responsables du gouvernement, l'auteur a bien pris le soin de déformer leurs noms ou leurs prénoms. Ce qui lui a permis une critique directe sans risque de censure.

${ }^{45}$ PREVOST, Guillaume. Op. cit. (Emplacements du Kindle 29). 
Soulevant ainsi des problèmes majeurs du quinquennat, à savoir le chômage, le déclassement, le déficit budgétaire, la crise économique au niveau de la politique intérieure de la France et l'intervention au Mali et en Centre Afrique et le problème des migrants au niveau de la politique étrangère, Prévost a pu afficher les erreurs commises par l'ex-président.

Quant à son deuxième héros, Michel Gravier, même avec son physique de François Hollande, il garde son statut d'homme du peuple, simple ouvrier d'EdenMaintenance. S'il arrive à résoudre le problème des ouvriers du nucléaire et les sauver du chômage, il n'est pas capable de faire face à beaucoup d'autres situations. Là aussi, l'auteur se sert de Gravier pour dessiner une image déformée du Chef de l'État. Ne l'avaitil pas accusé, à plusieurs reprises dans son roman, d'être incapable d'aligner que des banalités ? Ne l'avait-il pas surnommé le pingouin et le roi pingouin.

Nous pouvons donc déduire que Guillaume Prévost a pu tirer profit de l'exofiction, cette tendance qui autorise au romancier des inventions par l'écriture de dialogues et de monologues fictifs mais aussi par la représentation de périodes et des situations inconnues du grand public. À travers cette tendance, l'auteur a pu nous présenter un monde où la frontière entre le réel et la fiction est presque inexistante. Si l'auteure Iyonnaise Lucie Desbordes s'est servie de l'exofiction pour rendre hommage à son aïeule Marceline Desbordes-Valmore, poétesse française du $19^{\text {ème }}$ siècle, reconnue par les grands maîtres de la poésie romantique et symboliste, Prévost s'en sert pour dévoiler les défauts et les erreurs commises par l'ex-président français.

Même si Pierre Assouline, auteur de plusieurs biographies dont Simenon et Gaston Gallimard, et membre de l'Académie Goncourt depuis 2012, voit dans l'exofiction une certaine paresse de la part des romanciers, il faut admettre que Prévost a pu exploiter cette tendance pour réaliser son objectif. Selon Assouline, faire d'une personnalité ayant déjà existé, un personnage de roman est beaucoup plus simple et moins stressant pour le romancier que de créer son propre héros à partir du néant. Ceci donnera plus de chance au roman pour qu'il soit vendu plus facilement, "les millions de lecteurs et de Paris brûle-t- 
il savaient bien que Paris n'avait pas brûlé, et les spectateurs de Titanic que ça se terminait mal, et alors ? ${ }^{46}$, ajoute Assouline.

${ }^{46}$ ASSOULINE, Pierre, «L’exofiction, késako ? », La République des livres, 23 août 2016, [en ligne], consulté le 10 février 2017, tiré de http://larepubliquedeslivres.com/la-vie-des-autres/ 


\section{BIBLIOGRAPHIE}

\section{Référence de base :}

- PRÉVOST, Guillaume, Merci pour ce Roman, (French Edition), Éditions François Bourin, Septembre 2016, Édition du Kindle.

\section{Ouvrages :}

- $\quad$ CHALMIN, Philippe, La France de François Hollande, Chronique d'un quinquennat, (French Edition), Éditions François Bourin, 2017, Édition du Kindle.

- COTTA, Michèle, Le rose et le gris, Prélude au quinquennat de François Hollande, (French Edition), Édition Fayard, 2012, Édition du Kindle. ISBN : 9782-213-67536-7

- HANNA LOVE MOUKOUELLE, François Hollande, les raisons d'un échec, (French Edition), Mai 2018, Édition du Kindle.

- HOLLANDE, François, Les leçons du pouvoir, (French Edition), Éditions Stock, 2018, Édition du Kindle. ISBN : 978-2-234-08474-2

- TRIERWEILER, Valérie, Merci pour ce moment, Éditions des Arènes, Paris, 2014.

\section{Articles numériques et sitographie}

- $\quad$ AFP, «François Rebsamen enterre le CV anonyme », Le point, publié le 19/05/2015, [en ligne], consulté le 31 juillet 2017, tiré de https://www.lepoint.fr/societe/francois-rebsamen-enterrele-cv-anonyme-19-05-2015-1929465_23.php

- AFP, «Vallaud-Belkacem : "sans doute renforcer" la présence militaire française au Mali », L'express, publié le 04/11/2013, [en ligne], consulté le 23 juin 2017 tiré de https://www.lexpress.fr/actualites/1/societe/vallaud-belkacem-il-va-falloir-renforcer-lapresence-militaire-francaise-au-mali_1296495.html

- Anonyme, François Hollande : Les cinq raisons d'une impopularité record, le 06/11/2014, [en ligne], consulté le 26 février 2017, tiré de https://www.france24.com/fr/20141106francois-hollande-plus-bas-sondages-television-interview-impopularite-president-france

- ASSOULINE, Pierre, «L'exofiction, késako ? », La République des livres, 23 août 2016, en ligne, consulté le 10 février 2017, tiré de http://larepubliquedeslivres.com/la-vie-des-autres/

- DE CHALONGE, Mathilde, De la fiction à la biographie, l'exofiction, un genre qui brouille les pistes, dans $A L, 10.08 .2016$, https://www.actualitte.com/article/monde- 
edition/de-la-fiction-a-la-biographie-l-exofiction-un-genre-qui-brouille-lespistes/66392

- DUVAL, Guillaume, «Debout», Alternatives économiques, le 01/05/2016, [en ligne], consulté le 26 février 2017, tiré de https://www.alternatives-economiques.fr/guillaumeduval/debout/00011012

- ENVIEDECRIRE, Un roman peut-il impunément s'inspirer du réel ?17 mai 2011, https://www.enviedecrire.com/un-roman-peut-il-impunement-sinspirer-du-reel/

- GANDILLOT, Thierry, « Après l'auto, l'exofiction », Les Echos.fr, LE 24/08/2015, https://www.lesechos.fr/24/08/2015/LesEchos/22007-031-ECH_apres-1-auto--1exofiction.htm

- GUILLOT, Antoine, «Exofiction, késako ?», France culture, 04/09/2015, https://www.franceculture.fr/emissions/revue-de-presse-culturelle-d-antoineguillot/exofiction-kesako

- «La lutte antidiscrimination ou la promesse oubliée », Le Monde, Tribune, publié le 15 février 2013, [en ligne], consulté le 15 juin 2017, https://www.lemonde.fr/idees/article/2013/02/15/la-lutte-antidiscrimination-ou-lapromesse-oubliee 1833332 3232.html

- LEYRIS, Raphaëlle, « Rentrée littéraire : Le triomphe de l'exofiction », Le monde, publié le 16 août 2017, en ligne, consulté le 5 avril 2018, tiré de https://www.lemonde.fr/livres/article/2017/08/16/le-triomphe-de-lexofiction $51730673260 . \mathrm{html}$

- LOMBARDI, Rolland, « Le piteux bilan diplomatique du quinquennat de François Hollande », Le Figaro, publié le 07/09/2016, [en ligne], consulté le 23 juin 2017 tiré de http://www.lefigaro.fr/vox/monde/2016/09/06/3100220160906ARTFIG00124-le-piteux-bilan-diplomatique-du-quinquennat-defrancois-hollande.php

- $\quad$ LUI PRÉSIDENT, Les promesses de François Hollande passées au crible, [Site en ligne], consulté le 31 juillet 2018, tiré de https://www.luipresident.fr/francois-hollande/60engagements

- ODIBERT-TROIN, Olivier, «Migrants: La triple erreurs de François Hollande », Economie Matin, publié le 11/09/2015, [en ligne], consulté le 31 juillet 2018, tiré de http://www.economiematin.fr/news-migrants-francois-hollande-crise-politique

- POMMIERS, Eléa et DELRUE, Maxime «Emploi : Les modestes résultats du quinquennat Hollande », Le monde, le 27 avril 2017, [en ligne], consulté le 26 juin 2017, tiré de https://www.lemonde.fr/les-decodeurs/article/2017/04/27/emploi-etchomage-le-mediocre-bilan-du-quinquennat-hollande_5118948_4355770.html 
- $\quad$ SCAPPATICCI, Elena, « Audit de la Cour des comptes : Philippe juge « inacceptable » le dérapage des dépenses », Le Figaro, le 30/06/2017, [en ligne], consulté le 26 août 2017, tiré de http://www.lefigaro.fr/conjoncture/2017/06/29/20002-20170629ARTFIG00194audit-de-la-cour-des-comptes-sapin-et-eckert-se-justifient.php

- SLATE.FR, « Seulement en France », Hollande, Royal, Trierweiler et la presse étrangère, publié le 12 mai 2012, [en ligne], consulté le 30 juin 2017, tiré de https://www.slate.fr/lien/54905/hollande-royal-trierweiler-presse-etrangere

- Valérie TORANIAN, L'écrivain face au pouvoir, Revue des deux mondes, 20 AVRIL 2017, https://www.revuedesdeuxmondes.fr/lecrivain-face-pouvoir/\#mc4wp-form1

- VASSET, Philippe, cité par Frédérique ROUSSEL, « De passage secret », Libération, n 10039, 23 août 2013, [en ligne], consulté le 27 décembre 2017, URL : http://next.liberation.fr/livres/2013/08/22/philippe-vasset-de-passage$\underline{\text { secret_926385 }}$

- VERHAEGHE, Éric, « Fiscalité : une autre histoire du quinquennat Hollande » », Contrepoints, publié le 12 octobre 2016, [en ligne], consulté le 26 août 2017, tiré de https://www.contrepoints.org/2016/10/12/266654-fiscalite-croissance-histoire-quinquennat$\underline{\text { hollande }}$

\section{Vidéos}

- HOLLANDE, François, vidéo « L'intégralité du discours de François Hollande », Le Parisien TV, le $1^{\text {er }}$ décembre 2016, [en ligne], consulté le 26 août 2017, tiré de http://videos.leparisien.fr/video/l-integralite-du-discours-de-francois-hollande-01-12-2016x5469e1\#xtref=https\%3A\%2F\%2Fwww.google.fr\%2F

TRIERWEILER, Valérie, Interview, nouvelles déclarations choc à la BBC [VIDÉO], Mis à jour le 23/11/14, [en ligne], consulté le 31 juillet 2018, tiré de https://www.linternaute.com/actualite/personnalites/1205792-interview-trierweilernouvelles-declarations-choc-a-la-bbc-video/ 